\title{
Influence of magnetization on the applied magnetic field in various AMR regenerators
}

Mira, A.; de Larochelambert, T.; Espanet, C.; Giurgea, S.; Nika, P.; Bahl, Christian; Bjørk, Rasmus; Nielsen, Kaspar Kirstein

Published in:

Journal of Applied Physics

Link to article, DOI:

$10.1063 / 1.4986936$

Publication date:

2017

Document Version

Peer reviewed version

Link back to DTU Orbit

Citation (APA):

Mira, A., de Larochelambert, T., Espanet, C., Giurgea, S., Nika, P., Bahl, C., Bjørk, R., \& Nielsen, K. K. (2017). Influence of magnetization on the applied magnetic field in various AMR regenerators. Journal of Applied Physics, 122(13), [133901]. https://doi.org/10.1063/1.4986936

\section{General rights}

Copyright and moral rights for the publications made accessible in the public portal are retained by the authors and/or other copyright owners and it is a condition of accessing publications that users recognise and abide by the legal requirements associated with these rights.

- Users may download and print one copy of any publication from the public portal for the purpose of private study or research.

- You may not further distribute the material or use it for any profit-making activity or commercial gain

- You may freely distribute the URL identifying the publication in the public portal 


\title{
Influence of magnetization on applied magnetic field in various AMR regenerators
}

\author{
A. Mira, ${ }^{1}$ T. de Larochelambert, ${ }^{1, a}$ C. Espanet, ${ }^{1}$ S. Giurgea, ${ }^{1}$ P. Nika, ${ }^{1}$ C.R.H. Bahl, ${ }^{2}$ R. Bjørk, ${ }^{2}$ and \\ K.K. Nielsen ${ }^{2}$ \\ ${ }^{1}$ FEMTO-ST Institute, Univ. Bourgogne Franche-Comte, CNRS, Energy Department, Parc \\ technologique 2 avenue Jean Moulin, F-90000 Belfort Cedex, France. \\ ${ }^{2}$ Technical University of Denmark, Department of Energy Conversion and Storage, Ris $\phi$ Campus, \\ Frederiksborgvej 399, DK-40000 Roskilde, Denmark.
}

\begin{abstract}
:
The aim of this work is to assess the influence of a magnetic sample on the applied magnetic field inside the air gap of a magnetic circuit. Different magnetic sources including an electromagnet, a permanent magnet in a soft ferromagnetic toroidal yoke, as well as 2D and 3D Halbach cylinders are considered, using a numerical model. Gadolinium is chosen as magnetic material for the sample, due to its strong magnetocaloric properties and its wide use in magnetic refrigeration prototypes. We find that using uniform theoretical demagnetizing factors for cylinders or spheres results in a deviation of less than $2 \%$ in the calculation of internal magnetic fields at temperatures above the Curie point of gadolinium. Below the Curie point, a stronger magnetization of the cylinders and spheres leads to a larger deviation which can reach $8 \%$ when using uniform demagnetizing factors for internal magnetic field calculations. Published by AIP Publishing. https://doi.org/10.1063/1.4986936
\end{abstract}

\section{Keywords:}

Magnetization

Applied magnetic field

Demagnetization field

Permanent magnet working point

Magnetocaloric effect

\section{Nomenclature:}

$a$ regenerator plate length (m)

$a_{g} \quad$ air gap length (m)

\footnotetext{
${ }^{a}$ Author to whom correspondence should be addressed. Electronic mail: thierry.de-larochelambert@femto-st.fr
} 


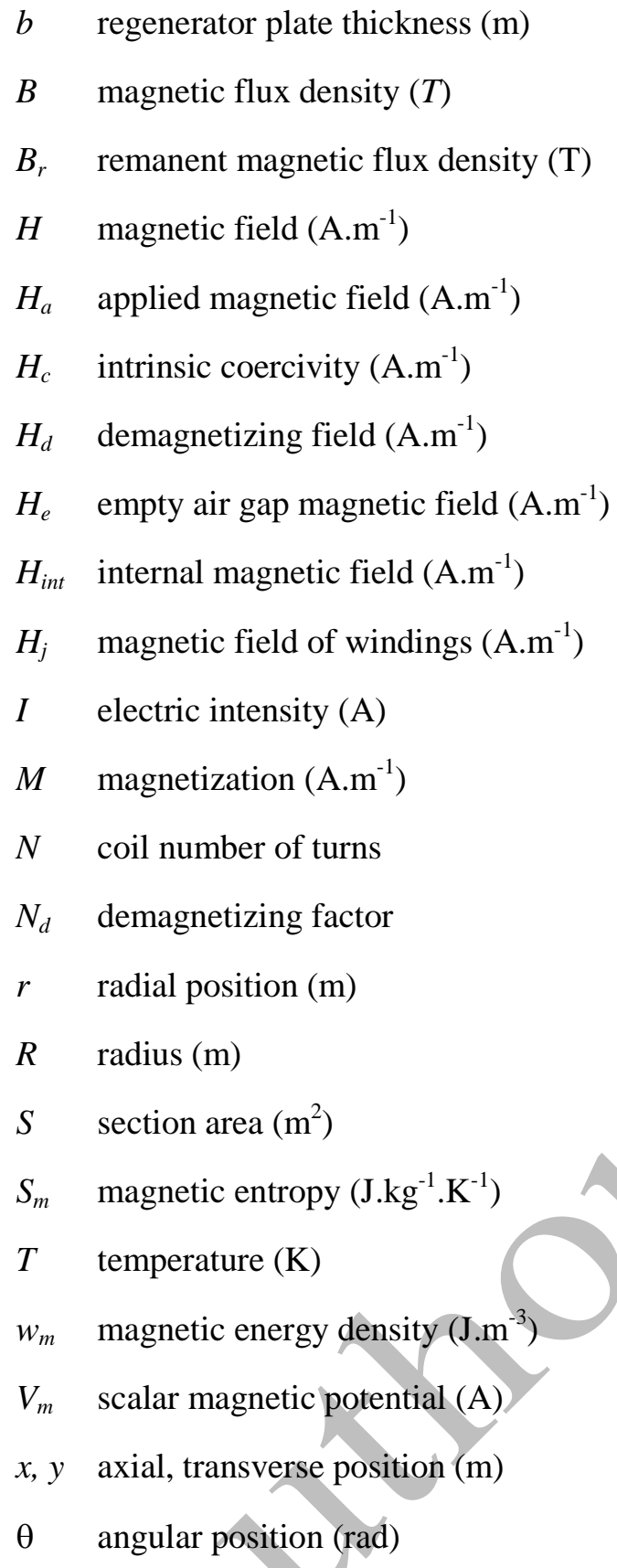

\section{Introduction}

The magnetocaloric effect (MCE) is the temperature change exhibited by magnetic materials submitted to a magnetic field change. ${ }^{1}$ Most of the magnetocaloric materials usually studied exhibit soft ferromagnetic properties. These materials are sorted according to the nature of their phase transition at their Curie temperature, in the vicinity of which the MCE is maximum. ${ }^{2}$ The MCE can be characterized both by the isothermal magnetic entropy change, $\Delta S_{m}$, and by the adiabatic temperature change, $\Delta T_{a d}$, which is the temperature increase of the magnetocaloric material when submitted to an 
applied magnetic field change under adiabatic conditions. The maximum $\Delta T_{a d}$ in pure $\mathrm{Gd}$ is about $4 \mathrm{~K}$ at $T_{c} \approx 293 \mathrm{~K}$ for a 0 to $1 \mathrm{~T}$ change in the magnetic field. Such high values of $\Delta T_{a d}$ have also been obtained in $f$ or $d$ metallic compounds. ${ }^{3}$

The adiabatic temperature change being only around a few kelvins in usual applied fields with permanent magnets, magnetocaloric materials are used as active materials in magnetocaloric devices for heating or cooling to produce higher temperature gradients. For this purpose, in order to transfer heat efficiently from cold source to hot sink in a cooling or heating device, the magnetocaloric materials are shaped as a regenerator (micro-plate or microspheres heat exchanger) that is crossed by an alternating flow, thus leading to so-called active magnetic regenerative (AMR) cycles ${ }^{4}$ that are widely used in experimental devices. A regenerator can be fundamentally considered as a porous medium through which a coolant fluid can alternatively flow. The thermal efficiency of such a regenerator depends on its specific surface area, on its geometric structure, on the thermo-physical properties of both the magnetocaloric material and the fluid, along with the flow characteristics.

Only few magnetocaloric device experiments use ordinary electromagnets to produce controlled high magnetic fields in large air gaps housing magnetocaloric regenerators, ${ }^{5,6}$ since the magnetic field production requires large inverters to feed the electromagnet with the necessary current, leading to prohibitive electric power consumption and low energy efficiency. However, electromagnets can be very useful in fundamental magnetocaloric test devices for time-controlled magnetization and heat exchange measurement in regenerators, ${ }^{7}$ since the magnetic field can thus be easily controlled.

For application and industrial development purposes, most experimental devices rather use permanent magnets as magnetic field sources for AMR cycles, by combining magnet or regenerator movements and alternating flow through regenerators, ${ }^{8}$ thus offering higher compactness and efficiency.

A central challenge in designing magnetocaloric devices is to correctly model the effect of the regenerator magnetization on (i) the whole magnetic field in electromagnets; (ii) the working point of permanent magnets (and therefore the available magnetic energy of magnetocaloric machines); (iii) the magnetic forces and torques undergone by the regenerators entering or leaving the air gaps in these machines.

The stray-field from the regenerator affects the applied magnetic flux density. An increasing number of papers have recently reported experimental and theoretical studies of the stray- and demagnetizing fields in magnetocaloric materials. Bahl and Nielsen ${ }^{10}$ measured the adiabatic temperature change $\Delta T_{a d}$ in gadolinium samples and sheets (purity 99.7\%) in up to $1.6 \mathrm{~T}$ applied magnetic fields with different orientations; they showed that the demagnetization field should be taken into account when reporting $\Delta T_{a d}$. Dai et al. ${ }^{11}$ used simplified formulae including the mean-field theory (MFT) parameters of gadolinium $B_{r}, H_{c}, \mu(H, T)$, leakage and reluctance factors to calculate the equivalent reluctance of equally spaced thin gadolinium sheets inserted into $\mathrm{NdFeB}$ magnetic circuits, in order to estimate their 
effect on the refrigeration power of a magnetocaloric machine. The average magnetic field in the gadolinium stack was calculated as a function of temperature for different geometries. The adiabatic temperature change was derived and found to be in good agreement with experimental results. Trevizoli et al. ${ }^{12}$ measured the adiabatic temperature change $\Delta T_{a d}$ in commercial-grade samples of gadolinium undergoing 0.03 to $1.65 \mathrm{~T}$ magnetic field changes with different orientations in order to obtain demagnetizing factors; they included their results in a 2D mathematical AMR model. This was used to quantify the temperature distribution in the parallel plate regenerator and thus to estimate the real cooling power. As a result, they concluded that the demagnetization field may induce important efficiency losses as high as $29 \%$, that can however be minimized with some geometry modification of the regenerator.

The demagnetizing field is closely related to both the local magnetization and the global shape of the magnetic sample. It is well-known that the magnetization $\vec{M}$ and demagnetizing field $\vec{H}_{d}$ vectors are uniform only in ellipsoids or infinite shapes with a uniform applied magnetic field $\vec{H}_{a} \cdot{ }^{13,14}$ The demagnetizing tensor $\overline{\bar{N}}_{d}$ of the sample, defined by

$$
\vec{H}_{d}=-\overline{\bar{N}}_{d} \cdot \vec{M}
$$

reduces to principal demagnetizing factors $N_{d x x}, N_{d y y}, N_{d z z}$ along the three principal axes of the magnetic sample when the demagnetizing field is uniform. In certain cases, such as a finite cylinder or rectangular prism, analytical expressions for average values (assuming a constant magnetization) of the demagnetization factors can be obtained. ${ }^{15-17}$ Techniques such as Fourier transforms and finite element methods can be used to accurately solve Maxwell's equations in order to calculate local demagnetizing field and factors in mágnetic material of arbitrary shape. ${ }^{16}$ Through the superposition principle, the effective internal local magnetic field inside the magnetic sample is expressed as

$$
\vec{H}_{i n t}=\vec{H}_{a}+\vec{H}_{d}
$$

In the case of magnetocaloric materials submitted to non-uniform applied magnetic fields, the solution of the thermal and magnetic equations requires numerical iterations, since the local magnetization $M\left(H_{i n t}, T\right)$ of the material closely depends on both temperature and the internal field. The local applied field depends on the magnetic circuit which in turn is modified by the stray field in its regenerative part, so that the problem is recursive. Moreover, phenomena such as magnetic saturation, flux leakages and other 3D effects may necessarily be included in the calculations for better accuracy.

In this paper, the magnetocaloric regenerators are supposed to be made of pure gadolinium. Demagnetization factors of the magnetocaloric samples do not need to be evaluated by theoretical formulae but instead result from precise calculation of the demagnetizing field in the whole sample, as presented hereunder (see section 2.1). 
Since any displacement of the working point of permanent magnets due to the magnetizationdemagnetization process in the magnetocaloric regenerator will impact the overall efficiency of a magnetocaloric device, this study aims at evaluating this displacement accurately in the case of magnetic circuits with permanent magnet, and comparing the results in common standard geometries for various shapes of gadolinium samples in 2D and 3D cases.

\section{Methodology}

\subsection{Magnetostatic model}

The following Maxwell's equations are solved by finite element method (FEM) in the magnetostatic case, using the commercial softwares Flux $2 \mathrm{D}^{\mathrm{TM}}$ and Flux3D ${ }^{\mathrm{TM}}:^{18}$

$$
\begin{gathered}
\overrightarrow{\operatorname{rot}}(\vec{H})=\vec{j} \\
\vec{H}=-\overrightarrow{\operatorname{grad}}\left(V_{\text {mred }}\right)+\vec{H}_{j} \\
\operatorname{div}\left(\mu_{0} \bar{\mu}_{r} \cdot \vec{H}+\vec{B}_{r}\right)=0
\end{gathered}
$$

where $\vec{H}_{j}, V_{\text {mred }}$ and $\vec{B}_{r}$ are the magnetic field produced by the windings only, the reduced scalar magnetic potential without windings and the remanent magnetic flux density, respectively.

After defining the magnetic and physical properties and geometries of each part of the system, automatic meshes are generated and refined in high flux density zones. A Dirichlet condition $V_{\text {mred }}=0$ is applied to the whole domain limit (infinite box). The above equations are solved for local magnetic field vector $\vec{H}$ at each node, which is identical to the internal magnetic field $\vec{H}_{i n t}$ inside the magnetic materials, hence accounting for demagnetizing field, non-linearities and magnetic saturation.

\subsection{Magnetic state modeling of gadolinium}

The magnetic behavior of gadolinium is introduced in the 2D and 3D Flux codes as a set of parametric $B\left(H_{\text {int }}\right)_{T}$ curves, calculated at each temperature $T$ from experimental data $M\left(\mu_{0} H_{a}, T\right)$ obtained in the G2Elab (Grenoble, France) with a high purity (> 99.9\%) sample. ${ }^{19}$

These data are corrected for the demagnetizing field using a critical thermodynamic model of the magnetic state of gadolinium, the best average demagnetizing factor $N_{d}$ being determined as a parameter along with other critical and magnetic parameters by means of least square methods and confirmed by analytical methods. ${ }^{20}$ The corrected magnetization curves $M\left(H_{\text {int }}, T\right)$ of gadolinium are 
then used to calculate all needed magnetic curves $B\left(H_{\text {int }}\right)_{T}$ which are then implemented as material data in the magnetostatic code:

$$
B\left(H_{\text {int }}\right)_{T}=\mu_{0} H_{\text {int }}+\mu_{0} M\left(H_{\text {int }}, T\right) .
$$

For the sake of simplicity, only two extreme values of temperature $T_{\text {hot }}=312 \mathrm{~K}$ and $T_{\text {cold }}=271 \mathrm{~K}$ around the Curie temperature $T_{c}=293 \mathrm{~K}$ of gadolinium are used for the simulated cases in this paper. Corresponding corrected magnetization curves are shown in Fig. 1, in adequacy with a lot of available data obtained for Gd in the related literature. ${ }^{21-25}$

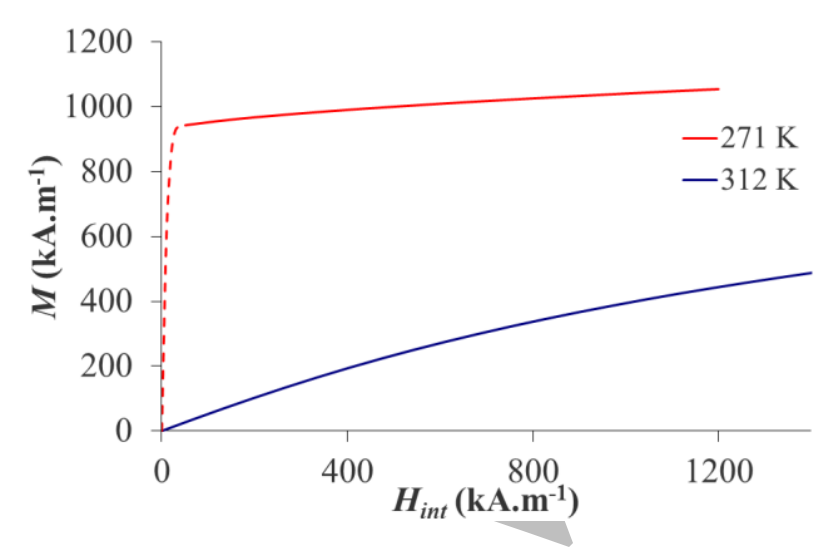

FIG. 1: Magnetization curves of gadolinium for hot and cold temperatures

\section{2D modeling of magnetic circuits with magnetized regenerators}

\subsection{Regenerator plate in electromagnet air gap}

We first consider a generic example of a plate of gadolinium in an air gap, the magnetic field being provided by an electromagnet. The general design of the system is shown in Fig. 2 and consists of a soft ferromagnetic toroidal yoke (NO Fe-Si M800-65A) with internal and external radii chosen as of $R_{i}$ $=131 \mathrm{~mm}$ and $R_{e}=171 \mathrm{~mm}$, and an air gap length $a_{g}=40 \mathrm{~mm}$. The magnetic field is produced by means of a 500 turns coil with a current of 100 A opposite the air gap. Obviously, this configuration is not optimal and induces significant magnetic flux losses, but it is simple and enables the production of a rather homogeneous field inside the air gap.

The regenerator in the center of the air gap consists of a single gadolinium rectangular plate of variable length $a$ and thickness $b$, and with a constant aspect ratio, $a / b$, of 19/3. This value was chosen for convenience in order to obtain a significant demagnetizing field and to keep the smallest thickness realistic. 


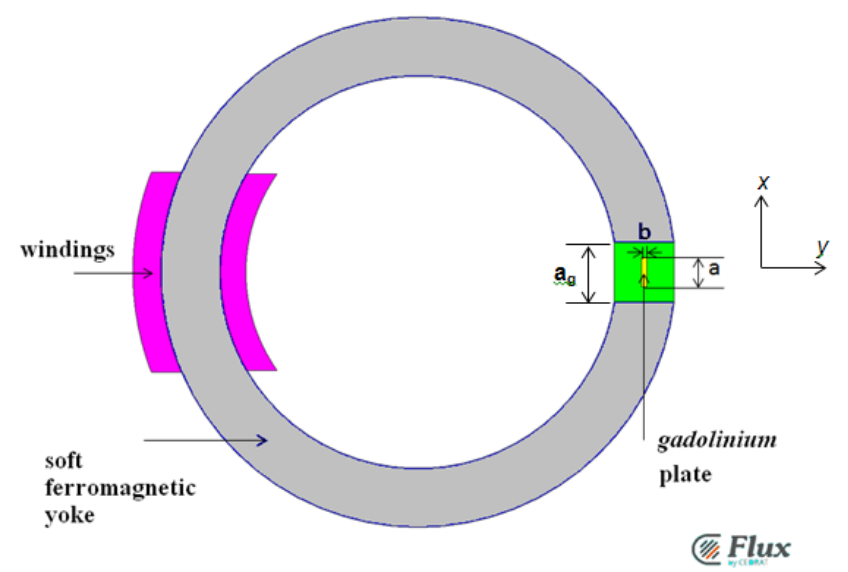

FIG. 2. 2D modeling of toroidal electromagnet (coil 500 turns, $I=100$ A). The air gap is shown in green, the magnocaloric plate in yellow and the windings in purple.

The axial magnetic field profiles calculated along the center line $x$ of the air gap with and without the gadolinium plate are shown in Fig. 3(a) at high temperature $T_{\text {hot }}=312 \mathrm{~K}$ (paramagnetic state of gadolinium) and in Fig. 3(b) at cold temperature $T_{\text {cold }}=271 \mathrm{~K}$ (ferromagnetic state of gadolinium). The axis origin coincides with the air gap center.

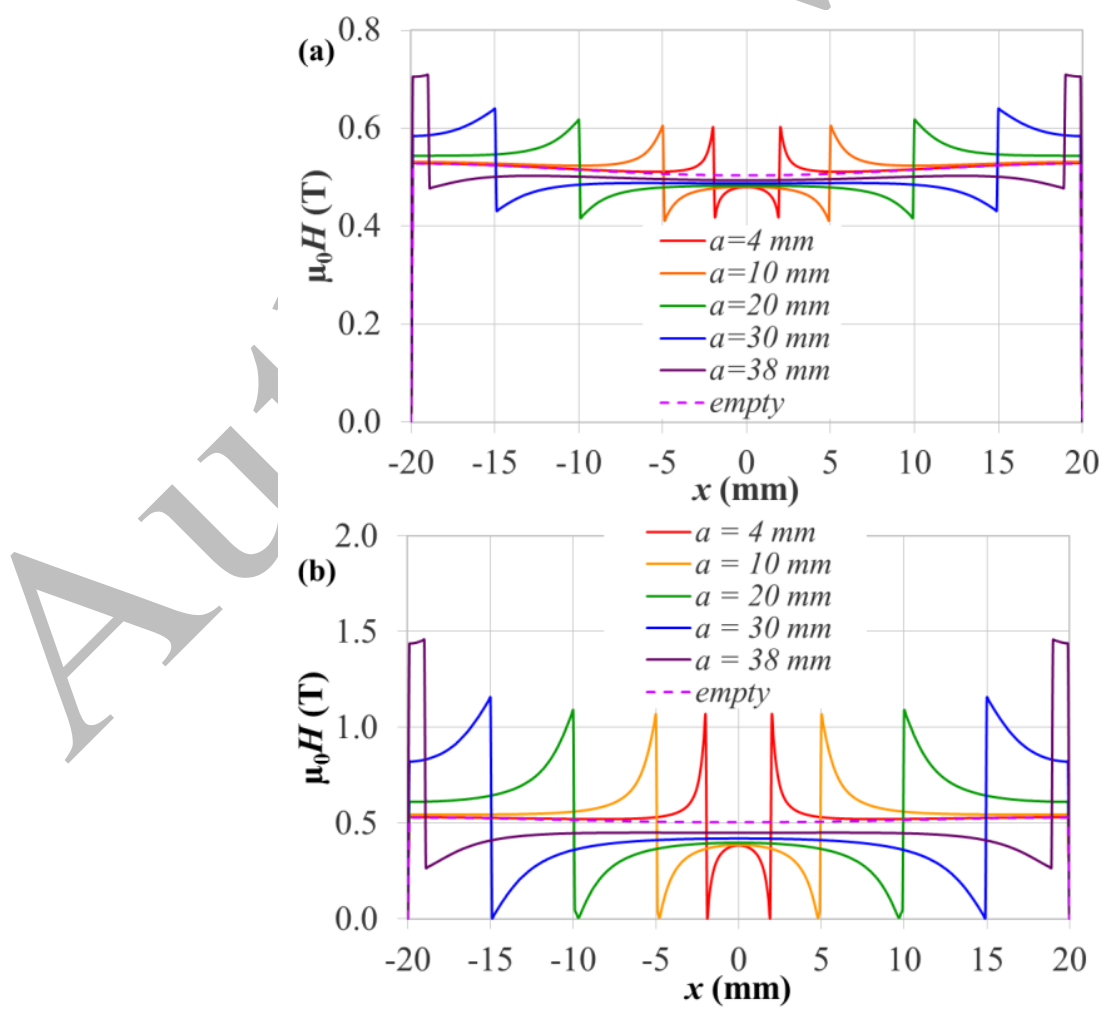

FIG. 3. Effect of gadolinium plate size on magnetic field in the electromagnet air gap.

(a) $T_{\text {hot }}=312 \mathrm{~K}$; (b) $T_{\text {cold }}=271 \mathrm{~K}$ 
It is observed for both temperatures that the magnetic field intensity in the air is higher in the presence of a regenerator plate for constant magnetomotive force $N I$, owing to a decrease in the air gap reluctance.

The enhancement of the magnetic field as well as its discontinuous drop at the edge of the regenerator are pronounced; this is due to both the magnetic permeability of gadolinium $\left(\mu_{r} \sim 1.5\right)$ and the demagnetizing field on the boundary surfaces, which increases the axial demagnetization factor $N_{d x x}$ close to the ends of the regenerator plate, as shown in Refs. 14 and 16.

Since the magnetization of gadolinium increases at lower temperatures, the demagnetizing field increases as well. As can be seen in Fig. 3(b), the steep change of $H$ across the frontiers of the gadolinium plate is about five times the one in Fig. 3(a), the demagnetizing factor being the same for each plate length. It must be kept in mind that the limit of the average demagnetization factor $N_{d z z}$ for infinite plate length $c$ is zero, $N_{d x x} \approx 0.168$ and $N_{d y y} \approx 0.832$ with aspect ratio $a / b=19 / 3$.

\subsection{Regenerator plate in air gap with permanent magnet source}

We now consider a case where the windings are removed and replaced by a permanent magnet as magnetic field source (Fig. 4). A high density grade NdFeB magnet has been chosen with remanence $B_{r}=1.43 \mathrm{~T}$ and intrinsic coercivity $H_{c}=1083 \mathrm{kA} \mathrm{m}^{-1}(1.36 \mathrm{~T})$ in order to obtain a sufficiently large magnetization of gadolinium and a higher sensibility to the demagnetizing field.

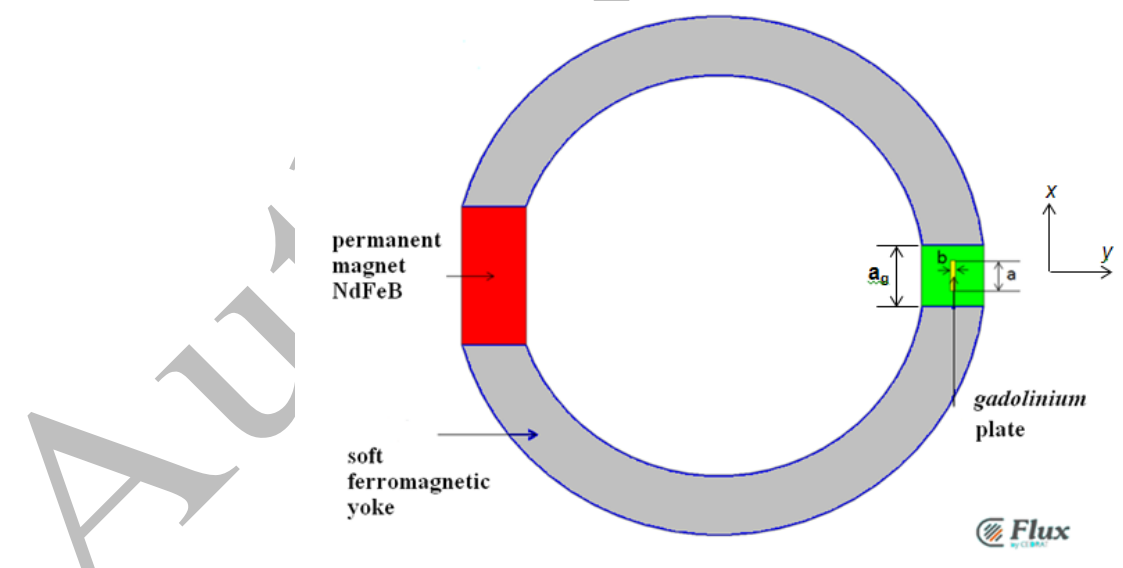

FIG. 4: 2D modeling of the magnetic circuit with permanent magnet

\subsubsection{Working point of a permanent magnet}

For a permanent magnet in a soft ferromagnetic yoke with an air gap, the magnetic energy density produced in the air gap is directly proportional to the magnetic energy density in the magnet. When introducing a magnetocaloric regenerator in the air gap, a change in $B$ and $H$ will take place in the whole magnetic circuit and modify the working point of the magnet and thus its energy density. 
The working point of a permanent magnet is usually defined as the coordinates along the easy axis $\left(H_{x}, B_{x}\right)$ of point $\mathrm{P}$ on the recoil curve representing the magnetic state of the magnet inside the magnetic circuit. It can also be characterized by the section-averaged energy density:

$$
w_{m}(P)=\frac{1}{2 S_{x}} \iint_{S_{x}}-B_{x} H_{x} d S_{x}
$$

Here, the working point $\mathrm{P}$ is placed on the recoil curve using the section-averaged flux density and magnetic field inside the magnet:

$$
\overline{B_{x}}(P)=\frac{1}{S_{x}} \iint_{S_{x}} B_{x} d S_{x} ; \overline{H_{x}}(P)=\frac{1}{S_{x}} \iint_{S_{x}} H_{x} d S_{x}
$$

The maximum magnetic energy density of the NdFeB magnet chosen for this simulation is $w_{m \text { max }} \approx$ $193.75 \mathrm{~kJ} \mathrm{~m}^{-3}$.

\subsubsection{Effect of temperature on the working point}

In order to determine the effect of the temperature of the gadolinium plate on the working point of the magnet, two series of simulations have been achieved for five sizes of the gadolinium plate, one at high temperature $T_{\text {hot }}=312 \mathrm{~K}$, the other at low temperature $T_{\text {cold }}=271 \mathrm{~K}$. The previous value of the aspect ratio $a / b=19 / 3$ was maintained.

As can be seen on Fig. 5, the longer the gadolinium plate, the lower the magnetic energy density $w_{m}$ of the magnet. The drop of energy density reaches $1.15 \%$ at $312 \mathrm{~K}$ (paramagnetic state of gadolinium) and $10.2 \%$ at $271 \mathrm{~K}$ (ferromagnetic state of gadolinium), which results from higher magnetization in cold gadolinium plates when introduced into the air gap for magnetization in AMR cycles, as shown in Fig.6.

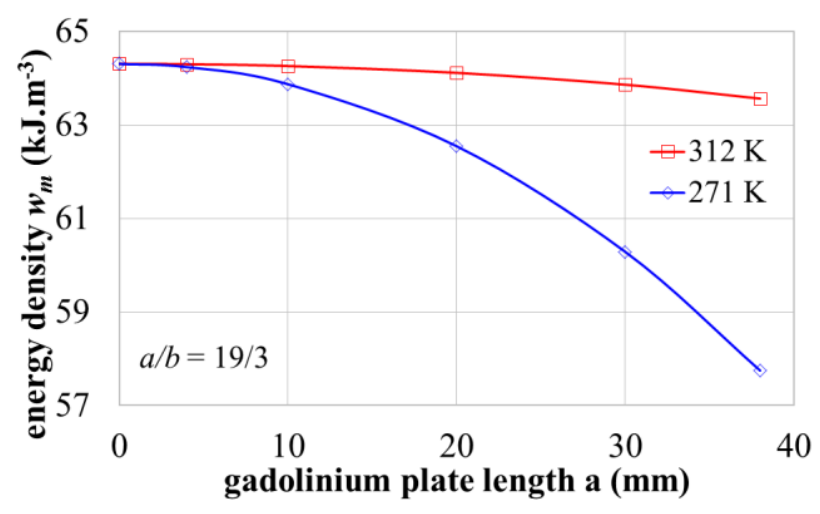

FIG. 5: Magnetic energy density of the permanent magnet with gadolinium plate in air gap 
When plotting the working point on the recoil curve of the magnet (Fig. 6), it appears that introducing the regenerator into the air gap results in a significant sweep of the working point towards the remanence, an effect that increases as the plate length increases or the temperature decreases. Therefore, the operating point of the magnet moves away from its initial position, so that the magnetic energy density $w_{m}=B H / 2$ becomes lower than its maximum value resulting in a loss in energy density.

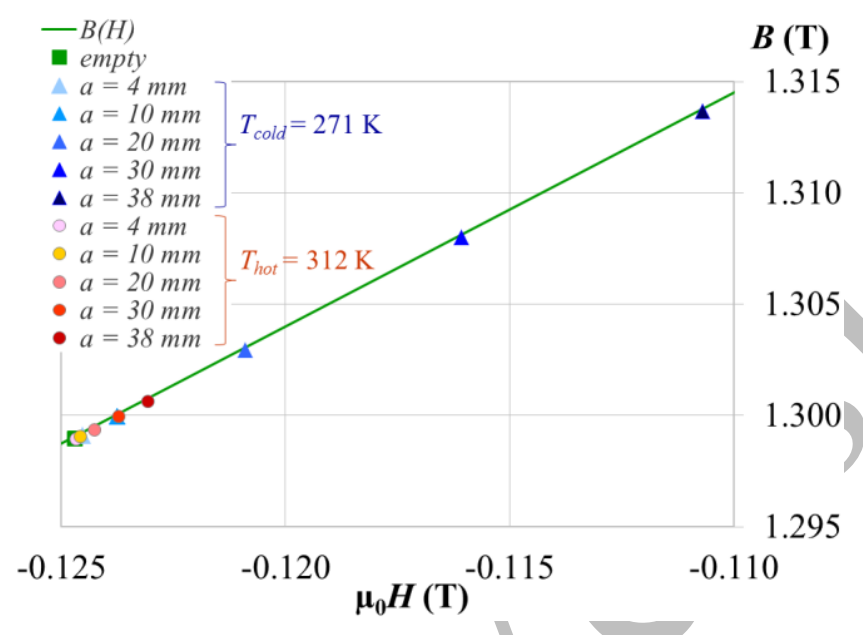

FIG. 6: Working point of permanent magnet for different plate lengths and temperatures (triangles: $T_{\text {cold }}=271 \mathrm{~K}$; circles: $T_{\text {hot }}=312 \mathrm{~K}$ )

This displacement is caused by the translation of the working line $(H, B)$ towards $H=0$ due to the magnetization $M$ of the gadolinium plate inside the air gap.

\section{2D modeling of Halbach cylinder}

We also consider the influence of gadolinium on the magnetic field produced by a Halbach cylinder, as this is a commonly used permanent magnet assembly for use in magnetic refrigeration. ${ }^{26}$ The considered Halbach cylinder is divided into 32 equivalent segments (index $p=-15$ to $p=16$ ) with different magnetization directions according to the following radial and azimuthal components:

$$
\left\{\begin{array}{l}
M_{r}^{\text {Halb }}=M^{\text {Halb }} \cos \left(p \frac{2 \pi}{32}\right) \\
M_{\theta}^{\text {Halb }}=M^{\text {Halb }} \sin \left(p \frac{2 \pi}{32}\right)
\end{array}\right.
$$

where $M^{\text {Halb }}$ is the remanent magnetization of the Halbach cylinder, so that the resulting magnetic field is almost uniform inside the whole inner air gap ( $1 \%$ drop in the range $-0.95<x / R_{i}<0.95$; max. $7.5 \%$ drop at $x / R_{i}= \pm 1$ ). The main characteristics of the Halbach cylinder are detailed in Tab. 1 . 
TABLE I

HALBACH CYLINDER CHARACTERISTICS

\begin{tabular}{lc}
\hline \multicolumn{1}{c}{ Dimensions } & Values \\
\hline External/inner radius $R_{e} / R_{i}$ & 1.75 \\
Number of segments & 32 \\
Remanent induction & $1.43 \mathrm{~T}$ \\
Radius of Gd discs $R / R_{i}$ & 0.10 .250 .50 .750 .95 \\
\hline
\end{tabular}

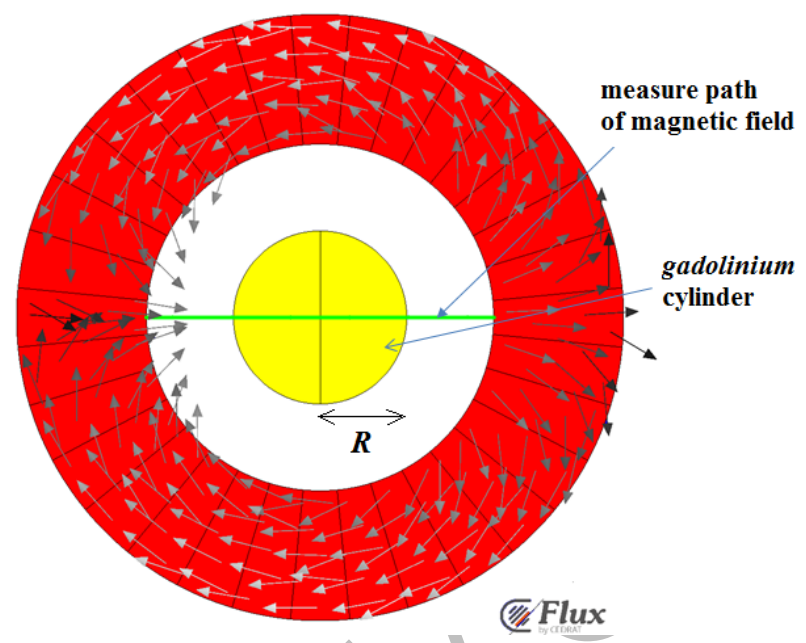

FIG. 7: 2D modeling of the Halbach cylinder with regenerator cylinders

\subsection{D Magnetic field in hot gadolinium cylinders inside Halbach cylinder}

The internal magnetic field $H_{\text {int } x}=H_{a p p x}-N_{d x x} M_{x}$ along the $x$ axis in hot gadolinium cylinders $\left(T_{h o t}=\right.$ $312 \mathrm{~K})$ of infinite length centered inside the Halbach cylinder has been calculated for different cylinder radii $R$ and compared to its value when the Halbach cylinder is empty (Fig. 7).

As expected, the internal magnetic field within the gadolinium cylinders is as homogeneous as the magnetic field in the empty Halbach cylinder, because of symmetry that in turn causes the magnetization $M$ and the demagnetization factors $\left(N_{d x x} \approx N_{d y y} \approx 1 / 2 ; N_{d z z} \approx 0\right)$ to be uniform as expected.

The demagnetizing field in the hot cylinders induces a drop of the internal field of around 19\% compared to the magnetic field $H_{e}$ in the empty Halbach cylinder. Even if this drop is a bit more pronounced for a smaller radius $R$, it remains quite similar for the different gadolinium cylinders.

However, to test the uniformity of the demagnetizing field and factor inside the gadolinium cylinders, the obtained internal magnetic field $H_{\text {int }}$ is compared to the standard internal magnetic field $H_{\text {int }}{ }^{\circ}=H_{e}$ $-M / 2$ that would be obtained if the magnetization of the cylinders were uniform in a uniform applied field $H_{a}=H_{e}$. The resulting curves $H_{\text {inn }} / H_{\text {int }}{ }^{\circ}$ are presented in Fig. 8. 


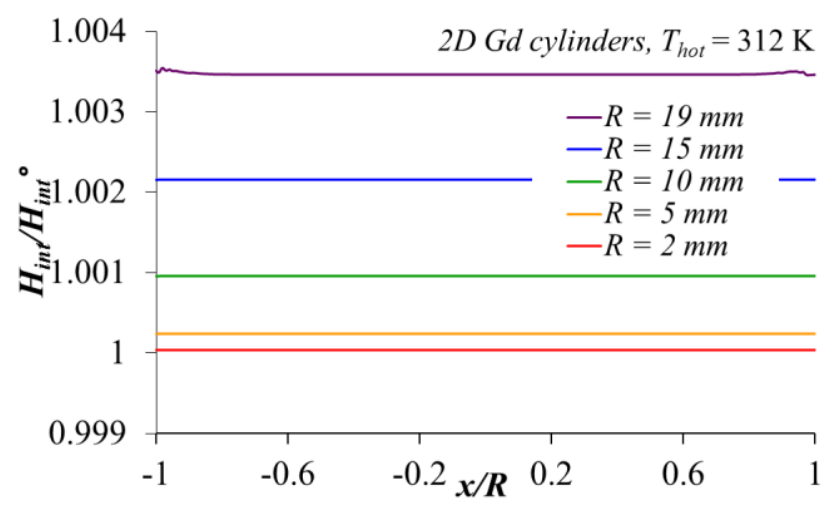

FIG. 8: Normalized magnetic field within 2D hot gadolinium cylinders in a 2D Halbach cylinder $\left(H_{\text {int }}{ }^{\circ}\right.$, standard internal magnetic field; $\left.T_{h o t}=312 \mathrm{~K}\right)$.

As expected in the $2 \mathrm{D}$ case, the internal field $H_{\text {int }}$ remains very close to the standard internal magnetic field and quite independent of the radius of the gadolinium cylinders.

As a consequence, the demagnetizing factor in 2D magnetocaloric cylinders inside the air gap of a 2D Halbach cylinder can be accurately assumed as uniform and equal to the theoretical value $N_{d}=1 / 2$ in the $x$ and $y$ directions. Furthermore, the internal magnetic field $H_{\text {int }}=H_{a}+H_{d}$ can be estimated from $H_{a} \approx H_{e}$ with great accuracy using $H_{d}=-N_{d} M$ with $N_{d}=1 / 2$.

\subsection{D Magnetic field in cold gadolinium cylinders inside Halbach cylinder}

The simulations have been conducted for the same gadolinium cylinders at cold temperature $T_{\text {cold }}=$ $271 \mathrm{~K}$ in the Halbach cylinder in order to evaluate the effect of higher demagnetization in ferromagnetic gadolinium on the magnetic field inside the Halbach cylinder.

The high magnetization of gadolinium in ferromagnetic state far below its Curie temperature causes the drop of internal magnetic field to be much more significant compared to the previous case. The decrease is around $76 \%$.

Again, it is interesting to test the uniformity of the demagnetizing field and factor inside strongly magnetized cold gadolinium cylinders in the ferromagnetic state, using the standard internal magnetic field $H_{\text {int }}{ }^{\circ}=H_{e}-M / 2$. The normalized curves $H_{\text {int }} / H_{\text {int }}{ }^{\circ}$ are shown on Fig. 9. 


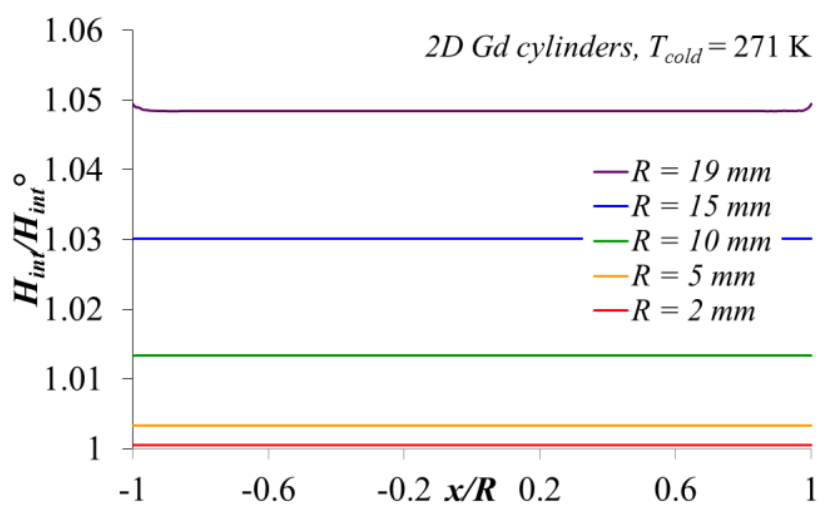

FIG. 9: Normalized magnetic field within 2D cold gadolinium cylinders in a 2D Halbach/cylinder $\left(H_{\text {int }}{ }^{\circ}\right.$, standard internal magnetic field; $\left.T_{\text {cold }}=271 \mathrm{~K}\right)$.

It is clear that the internal field $H_{\text {int }}$ remains close to the approximated internal field, with a relative difference ranging from $0.05 \%$ to about $5 \%$ as a function of the radius of the gadolinium cylinders.

Therefore, the theoretical demagnetizing factor $N_{d}=1 / 2$ and the applied magnetic field $H_{a} \approx H_{e}$ can be applied in 2D cold magnetocaloric cylinders inside the air gap of a 2D Halbach cylinder if their radius remains small compared to the inner radius of the Halbach cylinder. However, the discrepancy still remains weak $(<5 \%)$ even for large radii, so that this approximation can be considered as valid for $2 \mathrm{D}$ calculations in magnetocaloric cylinders in the uniform applied field produced by Halbach cylinders. In addition, the working point of the permanent magnets does not change significantly with the size of the gadolinium cylinder.

As a conclusion, the homogeneity of the internal magnetic field due to the symmetry of the system and the uniformity of the magnetic flux density does not seem to be significantly affected by the temperature, i.e. the magnetization, of the gadolinium, even for very small $R / R_{i}$ ratios, which is not the case for the gadolinium plates in magnetic circuits (Fig. 3(a) and 3(b)).

\section{3D modeling of Halbach cylinder}

The Halbach cylinder is now modeled with the code Flux $3 \mathrm{D}^{\mathrm{TM}}$ to account for $3 \mathrm{D}$ effects. In particular, its length $L$ is carefully chosen so that it is long enough $(L / R>>1)$ to generate a homogeneous magnetic field inside the air gap where a gadolinium sphere of various radii $R$ will be placed (Fig. 10).

For comparison, the external and internal radii $R_{e}$ and $R_{i}$ of the 3D Halbach cylinder are kept identical to the previous 2D simulation of the Halbach cylinder, and the $x$ axis is the main magnetic field direction inside air gap. Likewise, the different values of the gadolinium sphere radius $R$ are the same as those of the $2 \mathrm{D}$ gadolinium cylinders. 


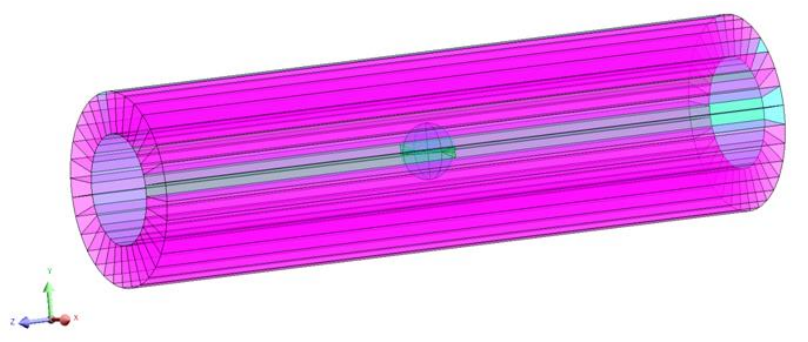

Flux

FIG. 10: 3D Halbach cylinder $\left(R_{e} / R_{i}=1.75 ; L / R_{i}=12.5\right)$ and gadolinium sphere model

Here again, the main purpose is to find the magnetic field and magnetization inside gadolinium spheres of different radii and to compare them to the case for which the theoretical value $N_{d}=1 / 3$ is uniformly imposed in the spheres.

\subsection{D magnetic field in hot gadolinium spheres inside a long Halbach cylinder}

We wish to examine the change in magnetic field inside the gadolinium sphere according to the sphere size, to determine the influence of the magnetization and demagnetizing field on the Halbach cylinder itself.

The internal magnetic field is very uniform, from about $87 \%$ of $H_{e}$ for the smallest radius, to $89 \%$ of $H_{e}$ for the largest sphere, $R / R_{i}=0.95$. It is less uniform in the orthogonal azimuthal directions, $\theta=$ $\pm \pi / 2$, where the magnetization inside the greatest sphere becomes sensitive to the small inhomogeneity of the applied magnetic field in the air gap very close to the inner side walls in these directions.

As also considered in the 2D case, in order to have more insight into the demagnetizing field and factor in the gadolinium spheres, it is of interest to normalize the internal magnetic field $H_{\text {int }}$ obtained in this 3D simulation by the standard internal magnetic field $H_{\text {int }}{ }^{\circ}=H_{e}-M / 3$. The result is presented in Fig. 11.

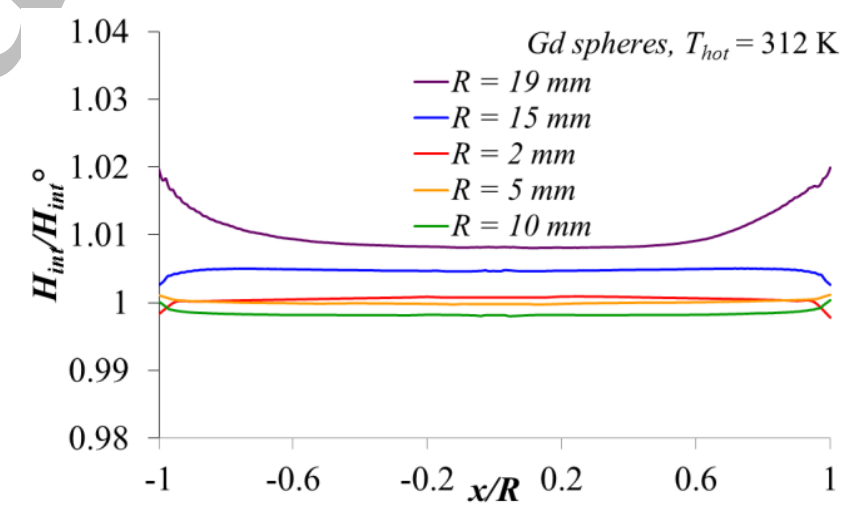

FIG. 11: Normalized magnetic field within hot gadolinium spheres in a 3D Halbach cylinder $\left(H_{\text {int }}^{\circ}\right.$, standard internal magnetic field; $\left.T_{\text {hot }}=312 \mathrm{~K}\right)$. 
As can be seen from the figure, the internal field $H_{\text {int }}$ remains very close ( 0 to $2 \%$ relative difference) to the approximate solution, and is independent of the size of the gadolinium sphere. Thus, the demagnetizing factor in magnetic spheres in the air gap of a Halbach cylinder can be correctly assumed to be uniform and equal to its theoretical value $N_{d}=1 / 3$ for all directions in the case of hot gadolinium spheres in paramagnetic state.

\subsection{D magnetic field in cold gadolinium spheres inside a long Halbach cylinder}

We consider the same setup as above, except with a cold temperature $T_{\text {cold }}=271 \mathrm{~K}$ of the gadolinium. As expected, the higher magnetization of the gadolinium sphere induces a larger decrease in the internal field, of around $50 \%$, for small radii.

By normalizing to the standard internal field, $H_{\text {int }}{ }^{\circ}=H_{e}-M / 3$, the validity of assuming a constant and homogeneous demagnetization factor can be tested. This is shown in Fig. 12.

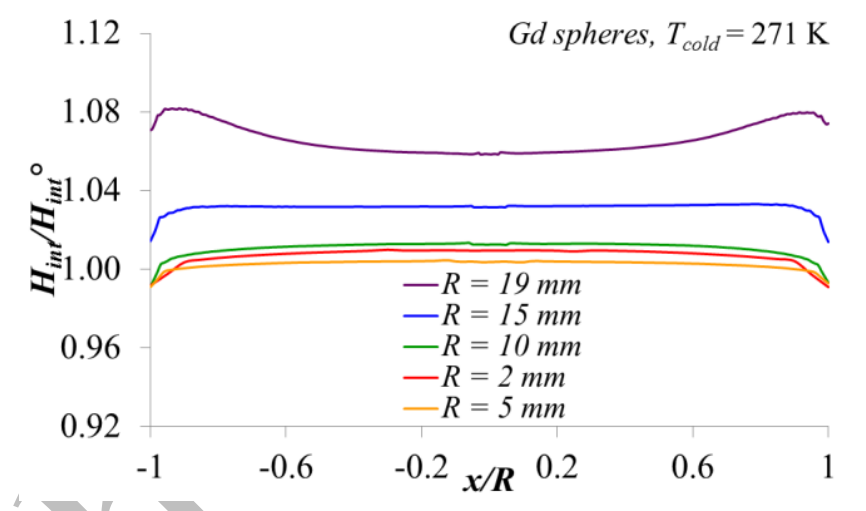

FIG. 12: Normalized magnetic field within cold gadolinium spheres in a 3D Halbach cylinder $\left(H_{\text {int }}^{\circ}\right.$, standard internal magnetic field; $\left.T_{\text {cold }}=271 \mathrm{~K}\right)$.

It can be observed that the difference between $H_{\text {int }}$ and the ideal case $H_{\text {int }}^{\circ}$ remains very small but slightly higher (up to $8 \%$ ) than for the high temperature, low magnetization gadolinium spheres. Therefore, the approximation of homogeneity for magnetization and demagnetizing factor is less correct in the case of cold gadolinium spheres than for cylinders.

Considering the results of the previous simulation of gadolinium plates inserted in a magnetic torus with permanent magnet (see section 3.2), the significant relative difference $\left(H_{\text {int }}-H_{\text {int }}{ }^{\circ}\right) / H_{\text {int }}{ }^{\circ}$ observed at low temperature can be interpreted by the following two coupled phenomena. Firstly, the small inhomogeneity of the magnetic field very close to the inside wall of the Halbach cylinder in orthogonal azimuthal directions $\theta= \pm \pi / 2$ results in amplified inhomogeneity in magnetization inside the spheres, although remaining small (lower than 8\%). Secondly, the stronger magnetization imposes the working point of the Halbach cylinder to move slightly towards the remanence. 
Thus, when good precision is required in predicting the performances of AMR cycles involving magnetocaloric spheres as regenerators moving into or out of Halbach cylinders, simply applying a uniform demagnetization factor $N_{d}=1 / 3$ may not completely match this objective during phases where the magnetocaloric spheres are submitted to high magnetic fields at temperatures below their Curie temperature.

\section{Conclusion}

To assess the influence of magnetocaloric regenerators on the magnetic field inside magnetic circuits, various situations have been simulated considering electromagnets, permanent magnets and Halbach cylinders interacting with gadolinium plates, cylinders and spheres in 2D and 3D computations. The major role of the temperature has been highlighted in each case, because of the strong change in the magnetic state of gadolinium around its Curie temperature.

In electromagnet circuits, the introduction of magnetic plates into the air gap leads to a significant increase of the magnetic field in the air gap induced by the reluctance decrease, as well as a drop of the internal magnetic field inside the plates due to a non-uniform demagnetizing field. These modifications are stronger at low temperature since the magnetization is higher. Therefore, when using electromagnets in experimental test benches for magnetocaloric applications, particular attention must be paid to the important change in the air gap reluctance while moving magnetic materials of various geometries into the latter. The large modification of the magnetic field requires complete calculations of the whole magnetic circuit for good precision of the magnetocaloric properties and refrigeration performances in AMR cycles. Moreover, assuming uniform demagnetization factors in rectangular regenerator plates may lead to erroneous estimations in magnetocaloric effect.

In permanent magnet circuits, introducing magnetic plates causes the working point of the magnet to move towards the remanence, leading to a significant magnetic field and magnetic energy density decrease of the permanent magnet that is more pronounced at temperatures below the Curie temperature. The change in magnetic energy density of the magnet may reach more than $10 \%$ and the change in magnetic field or flux density more than $1 \%$, when introducing cold magnetocaloric plates, depending on the size of the magnetocaloric samples.

When using Halbach magnets, good homogeneity of the magnetic field can be reached, inducing an almost homogeneous magnetization and demagnetizing field inside magnetocaloric cylinders. As expected, the drop of internal magnetic field is significant at low temperature and coherent with a uniform demagnetizing factor $N_{d}=1 / 2$ for magnetocaloric cylinders and $N_{d}=1 / 3$ for magnetocaloric spheres. 
Therefore, simply applying uniform theoretical demagnetizing factors for cylinders or spheres offers good enough precision (less than $2 \%$ deviation) in calculation of applied and internal magnetic fields at temperatures above the Curie point of the magnetocaloric material.

However, temperatures below the Curie point induce stronger magnetization of the magnetocaloric cylinders and spheres, which, along with a small inhomogeneity, can lead to less precision (up to 8\%) when using uniform demagnetizing factors for applied and internal magnetic field calculations. This should be taken into account for the sake of precision when calculating the MCE or the AMR cycle efficiencies.

The results given above indicate that this discrepancy could reach some percent for magnetic field, leading therefore to some uncertainty in the estimation of MCE and for AMR efficiencies.

As a conclusion, the design of magnetic systems for magnetocaloric applications such as magnetic refrigeration, air conditioning and heat pumping requires careful attention to the change in magnetic field and working point of permanent magnets when magnetocaloric regenerators move into or out of air gaps.

\section{References}

${ }^{1}$ A. Smith, C.R.H. Bahl, R. Bjørk, K. Engelbrecht, K.K. Nielsen and N. Pryds, "Materials challenges for high performance magnetocaloric refrigeration devices", Advanced Energy Materials 2, 1288-1318 (2012)

${ }^{2}$ A.M. Tishin, in Handbook of Magnetic Materials, edited by K.H.J. Buschow (Elsevier, 1999), Vol. 12, pp. 395524.

${ }^{3}$ V.K. Pecharsky, K.A. Gschneider, Y. Mudryk, D. Paudyal, "Making the most of the magnetic and lattice entropy changes", J. Magn. Magn. Mat. 321 (2009) 3541-3547.

${ }^{4}$ G.V. Brown, "Magnetic heat pumping near room temperature”, J. Appl. Phys. 47 (1976) 3673.

${ }^{5}$ B.F. Yu, Q. Gao, B. Zhang, D.X. Yang, Y. Zhang, "Experimental investigation on refrigeration performance of a reciprocating active magnetic regenerator of room temperature magnetic refrigeration", in Proceedings of the $1^{\text {st }}$ International Conferencé on Magnetic Refrigeration at Room Temperature, Montreux, Switzerland, 27-30 September 2005, pp. 375-391.

${ }^{6}$ A.A. Coelho, S. Gama, A. Magnus, G. Carvalho, "Prototype of a Gd-based rotating magnetic refrigerator for work around room temperature", Proceedings of the $3^{\text {rd }}$ International Conference on Magnetic Refrigeration at Room Temperature, Des Moines, Iowa, USA, 11-15 May 2009, pp. 381-386.

${ }^{7}$ A. Mira, C. Espanet, T. de Larochelambert, S. Giurgea, P. Nika, "Influence of computing magnetic field on thermal performance of a magnetocaloric cooling system", European Journal of Electrical Engineering 17(3-4) (2014) 151-170.

${ }^{8}$ B. Yu, M. Liu, P.W. Egolf, A. Kitanovski, “A review of magnetic refrigerator and heat pump prototypes built before the year 2010”, Int. J. Refr. 33 (2010) 1029-1060.

${ }^{9}$ F. Allab, A. Kedous-Lebouc, J.P. Yonnet, J.M. Fournier, "A magnetic field source system for magnetic refrigeration and its interaction with magnetocaloric material”, Int. J. Refr. 29 (2006) 1340-1347.

${ }^{10}$ C.R.H. Bahl, K.K. Nielsen, "The effect of demagnetization on the magnetocaloric properties of gadolinium", J. Appl. Phys. 105, 013916 (2009). 
${ }^{11}$ W. Dai, B.G. Shen, D.X. Li, Z.X. Gao, “Application of high-energy NdFeB magnets in the magnetic refrigeration”, J. Magn. Magn. Mat. 218 (2000) 25-30.

${ }^{12}$ P.V. Trevizoli, J.R. Barbosa Jr, P.A. Oliveira, F.C. Canesin, R.T.S. Ferreira, “Assessment of demagnetization phenomena in the performance of an active magnetic regenerator”, Int. J. Refr. 35 (2012) 1043-1054.

${ }^{13}$ J.S. Osborn, "Demagnetizing factor of the general ellipsoid”, Phys. Rev. 67 (1967) 351-357.

${ }^{14}$ R.I. Joseph, E. Schlömann, “Demagnetizing field in non-ellipsoïdal bodies”, J. Appl. Phys. 36 (1965) 1579.

${ }^{15}$ A. Aharoni, "Demagnetizing factors for rectangular ferromagnetic prisms", J. Appl. Phys. 83 (1998) 34323434.

${ }^{16}$ A. Bagnérés, S. Durbiano, “3D computation of the demagnetizing field in a magnetic material of arbitrary shape”, Comp. Phys. Comm. 130 (2000) 54-74.

${ }^{17}$ R. Bjørk, C. R. H. Bahl, "Demagnetization factor for a powder of randomly packed spherical particles", Appl. Phys. Lett. 103 (2013) 102403pl

${ }^{18}$ Cedrat, Guide d'utilisation Flux11, Grenoble, France (2012).

${ }^{19}$ F. Allab, A. Kedous-Lebouc, J.M. Fournier, J.P. Yonnet, Numerical modeling for active magnetic regenerative refrigeration, IEEE Trans. Magn. 41 (10) 2005, 3757-3759.

${ }^{20}$ T.de Larochelambert, "Looking for a magnetic equation of state of gadolinium" (to be published).

${ }^{21}$ F. Döbrich, J. Kohlbrecher, M. Sharp, H. Eckerlebe, R. Birringer, A. Michels, "Neutron scattering study of the magnetic microstructure of nanocrystalline gadolinium”, Phys. Rev. B 85 (2012) 094411.

${ }^{22}$ M.N. Deschizeaux, G. Develey, "Equation magnétique d'état du gadolinium au voisinage du point de Curie", Le Journal de Physique 32 (1971) 319-323.

${ }^{23}$ C.D. Graham Jr, Magnetic behavior of gadolinium near the Curie point, J. Appl. Phys. 36 (1965) 1135-1136.

${ }^{24}$ J.F. Elliott, S. Legvold, F.H. Spedding, "Some magnetic properties of gadolinium metal”, Phys. Rev. 91 (1953) 28-30.

${ }^{25}$ H.E. High, S. Legvold, F.H. Spedding, "Magnetization and electrical resistivity of gadolinium single crystals", Phys. Rev. 132 (1963) 1092-1097

${ }^{26}$ R. Bjørk, C.R.H. Bahl, A. Smith, N. Pryds, "Review and comparison of magnet designs for magnetic refrigeration", Int. J. Refr. 33 (2010) 437-448. 